\title{
Recreational Drugs and Sexual Behavior in the Chicago MACS/CCS Cohort of Homosexually Active Men
}

\author{
David G. Ostrow $(1,2)$ \\ Eugenio D. Beltran (1) \\ Jill G. Joseph (1) \\ Wayne DiFranceisco (1) \\ Jerry Wesch $(3,4)$ \\ Joan S. Chmiel $(4,5)$ \\ University of Michigan and the Coping and Change Study (1) \\ AIDS Psychobiology Program, University of Michigan Medical School (2) \\ Howard Brown Health Center of Chicago (3) \\ Chicago Multicenter AIDS Cohort Study (4) \\ Cancer Center Biometry Section, Northwestern University Medical School (5)
}

\begin{abstract}
Since initial reports emerged of an association between recreational drug use and high-risk sexual behaviors in gay men, there has been interest in studying this relationship for its relevance to behavioral interventions. Reported here are the longitudinal patterns of alcohol and recreational drug use in the Chicago Multicenter AIDS Cohort Study (MACS)/Coping and Change Study (CCS) of gay men. A pattern of decreasing drug use over 6 years was observed that paralleled a decline in high-risk sexual behavior (i.e., unprotected anal intercourse). In contrast, alcohol consumption tended to be more stable over time, and to show no relationship to sexual behavior change. Men who combined volatile nitrite (popper) use with other recreational drugs were at highest risk both behaviorally and in terms of human immunodeficiency virus-1 (HIV) seroconversion throughout the study. Popper use also was associated independently with lapse from safer sexual behaviors (failure to use a condom during receptive anal sex). Use of other recreational substances showed no relationship to sexual behavior change patterns, and stopping popper use was unrelated to improvement in safer sexual behavior. When popper use and lapse from saler sex were reanalyzed, controlling for primary relationship status, popper use was associated with failure to use condoms during receptive anal sex among nonmonogamous men only. These findings suggest an association between popper use and high-risk sexual behavior among members of the Chicago MACS/C.CS cohort that has relevance to HIV prevention intervention efforts.
\end{abstract}

This work was supported by funding from the National Institute of Mental Health

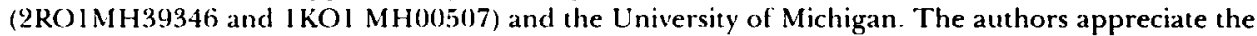
contribution of biomedical data by the Chicago Multicenter AIDS Cohort Study (AI-32535 from the National Institute of Allergy and Infectious Diseases). We are grateful to the staff of the Howard Brown Health Center of Chicago for assistance in data collection and to the men of the Coping and Change Study for their participation in this research.

Correspondence and requests for reprints should be sent to David Ostrow, Medical College of Wisconsin, Community Health Behavior Program, 1201 N. Prospect Ave., Milwaukee, WI 53202. 


\section{INTRODUCTION}

The concentration of early cases of acquired immunodeficiency syndrome (AIDS) among homosexually active men living in major urban centers in the United States led to an interest in sexual behavior and its role in the transmission of pathogens causing immune suppression. From the earliest case control studies conducted by the Centers for Disease Control's (CDC) Task Force on Kaposi's Sarcoma and Opportunistic Infections (Jaffe et al., 1983) to recent studies of predictors of human immunodeficiency virus-type 1 (HIV) infection (Penkower et al., 1991), recreational psychoactive drug use has been associated with HIVrelated illness or infection among homosexual men. However, the nature of this association has been controversial. Stall, McKusick, Wiley, Coates, and Ostrow (1986) first demonstrated a significant association between recreational drug use and levels of HIV-transmitting sexual behaviors (predominantly unprotected anal intercourse) in the San Francisco Men's Health Study (SFMHS) cohort, a finding since replicated in other longitudinal studies (Calzavara et al., 1993; Doll et al., 1989; Martin, Dean, Garcia, \& Hall, 1988; Ostrow et al., 1990). In contrast, several longitudinal studies have either failed to find an association between recreational drug use and patterns of sexual behavior change (McCusker et al., 1990) or reported a weakening of any associations over time (Martin, 1990). In addition, several studies that have contrasted specific homosexual encounters with and without reported recreational substance use (critical event analysis) have failed to find a higher likelihood of unprotected anal intercourse when recreational substances such as alcohol were involved (Gold, Skinner, Grant \& Plummer, 1991; Weatherburn et al., 1993). These conflicting findings have led some to question the validity of any relationship between substance use and high-risk sex among homosexual men (Weatherburn, 1992), at least to question the role of alcohol intoxication. Others have suggested that coincident risktaking behaviors rather than direct drug effects on sexual behavior may explain the observed associations (Leigh, 1990).

In light of the contradictory findings to date, it becomes important to continue evaluating the long-term associations between drug and/or alcohol use, and high-risk sexual behavior. In this study, the patterns of recreational drug use and their relationship to sexual behavior change and seroconversion over a 6-year period (April 1984-December 1990) were examined among men participating in both the behavioral/psychosocial Coping and Change Study (CCS) and the Chicago component of the Multicenter AIDS Cohort Study (Chicago MACS). The current study extended the prior cross-sectional observations with this cohort, to explore in a prospective longitudinal manner the patterns of drug use over time and their possible association with changes in a specific high-risk sexual behavior-unprotected anal intercourse. Because these longitudinal observations confirmed our earlier cross-sectional findings of an association between the use of volatile nitrites (poppers) and unprotected receptive anal sex (RAS), further analyses focused on the nature of that association. In particular, the following set of questions was crucial to understanding the na- 
ture of prior observations of increased sexual risk taking by the men in this cohort reporting recreational substance use: (a) How specific was the association in terms of type and number of substances used and was the sexual risk taking in a monogamous relationship or with a casual partner?; (b) Was initiation of specific substance use associated with increased risk of failure to use condoms (lapse to unsafe sex)?; and (c) Was stopping the use of a recreational substance associated with subsequent reductions in unprotected anal sex (remission to safer sex)?

\section{MATERIALS AND METHODS}

\section{Study Population}

The study population consisted of homosexual men participating in both the Chicago MACS, which collected drug use and physical health data at each semiannual visit, and the CCS, which collected self-reported mental health, sexual behavior, and psychosocial data. The general characteristics of the MACS and its study populations are described elsewhere (Chmiel et al., 1987; Kaslow et al., 1987), as are the objectives and design of the CCS (Joseph et al., 1984). The men were recruited for the Chicago MACS through either a gay community-based sexually transmitted disease clinic (the Howard Brown Health Center) or the Infectious Disease Program of Northwestern University Medical School. At the time of the baseline MACS assessment (April 1984-March 1985), men were invited to participate in the CCS. Those agreeing to do so were asked to complete a take-home questionnaire to be returned by mail 2 weeks following their MACS baseline assessment and semiannual follow-up visits. Of the 1,102 Chicago MACS participants who completed the drug use and physical health assessments, 1,005 also participated in the CCS, although only 381 men completed all nine MACS and CCS assessment visits and questionnaires between mid-1984 and the end of 1990. Attrition was most often due to incomplete participation rather than to disenrollment from the MACS or CCS. As described later, there were no statistically significant differences in either drug use or specific sexual behaviors in the group of men participating consistently and the larger group participating inconsistently.

\section{Alcohol and Drug Use Measures}

Alcohol and drug use measures were collected as part of the face-to-face interviews conducted at each semiannual MACS visit. At baseline, questions were asked to determine the frequency (but not quantity) of use of each of 10 classes of recreational drugs during the past 2 years and during the past 6 months. For alcoholic beverages, both frequency and quantity were recorded. At all follow-up visits, the same questions were repeated but only for the period since the last visit (usually 6 months).

From these data, summary substance use measures were created as follows: (a) Frequency of use of each drug and summation of all reported drug use in the 
prior 6 months; (b) number of alcoholic beverages consumed per month, further classified using National Institute of Alcoholism and Alcohol Abuse (NIAAA) categories into light (fewer than 13 drinks/months), moderate (13-59 drinks/month), or heavy (more than $60 \mathrm{drinks} /$ month) (Clark \& Midanik, 1982); and (c) a four-level drug use index based on an earlier study of predictors of high-risk sexual behavior at baseline among the entire four MACS cohorts (Ostrow et al., 1990): Level 1, no recreational drugs during the past 6 months; Level 2 , one or more recreational drugs but no poppers; Level 3, poppers and up to two other recreational drugs; and Level 4, poppers plus three or more recreational drugs.

\section{Sexual Behavior Measures}

The sexual behavior measures contained in the CCS questionnaire inquired about number of four different types of sexual partners in the past month (primary, steady, casual, and anonymous), the actual number of three different sexual activities (receptive oral, receptive anal, and insertive oro-anal ["rimming"] intercourse), and the consistency with which condoms were used for oral and anal intercourse. Beginning with Visit 4 , additional questions were asked regarding the frequency of insertive anal intercourse and use of condoms in the past month. From the partner numbers and receptive anal intercourse responses, each participant was classified into one of four sexual risk index levels for each visit (Ostrow, 1989): (a) celibate (zero partners), (b) low-risk, defined as RAS only with primary partner and always using a condom, (c) modified highrisk, defined as unprotected RAS with only one partner and/or consistently protected RAS with multiple partners, and (d) high-risk, defined as unprotected RAS with multiple partners.

Prior studies have reported on the validity of the sexual risk index as a predictor of seroconversion for this cohort (Joseph et al., 1987; Ostrow et al., 1989). For the current analyses, both the sexual and drug use indices were examined for their ability to predict relative rates of seroconversion across the $4^{1 / 2}$-year study period (1984-1988). Person-year seroconversion rates were obtained for the cohort, stratified into the four levels of the behavioral index of interest (i.e., sexual risk index, drug use index, and their interaction) at each visit. The number of seroconversions that occurred hetween visits was obtained for each stratum from MACS serological data (Detels et al., 1989). The number of seroconverters (numerator) and persons at risk of seroconversion (seronegatives at beginning of interval, denominator) were then accumulated across the eight semiannual intervals for each behavioral category and the results reported as seroconversions per 100 person-years of observation.

\section{Data Analysis}

Two sets of analyses were performed: (a) using the maximum number of participants for each visit (maximum number analyses), and (b) using only the 
subsample of participants assessed at all nine visits (panel analyses). Because no differences in rates of either RAS or drug use were found in the two sets of analyses, descriptive data based on the maximum number of participants available at each visit are presented here. For prospective analyses of associations between specific patterns of substance use and changes in sexual risk taking, only participants completing both semiannual pairs of study assessments were examined.

Bivariate analyses included two sample $t$ test and chi-square procedures. When the two variables of interest were dichotomous, odds ratios (ORs) and their $95 \%$ confidence intervals $(95 \% \mathrm{CI})$ were used as the preferred measures of association. Nonparametric statistics were used when variables were counts or showed strong indication of deviation from a Gaussian distribution. Kendall tau (Sprent, 1989) and Spearman rho were used to analyze correlations between variables of interest. In order to test associations between change in pattern of use of a specific drug and the adoption or cessation of safer sexual practices (condom use during insertive or receptive anal intercourse), $2 \times 2$ tables were used and ORs with $95 \%$ CIs reported.

\section{RESULTS}

\section{Alcohol and Recreational Drug Use Patterns, 1984-1990}

Alcohol was reportedly used by 90 to $95 \%$ of participants at each visit. Between 12 and $16 \%$ of the participants reported moderate alcohol consumption, and heavy alcohol use declined from $28 \%$ at baseline to 12 to $15 \%$ thereafter. The percentage of nonusers in the cohort increased gradually from 5 to $10 \%$ during the study period. No significant cross-sectional differences in rates of unprotected sex were observed among groups based on alcohol consumption except for heavy users at baseline who reported a higher frequency of unprotected RAS than did nonusers or moderate users $(p<.05)$. This association was not observed at any subsequent period.

Marijuana and volatile nitrites (poppers) were the drugs most frequently used by participants throughout the study period. Participants reported a decrease in use of both drugs from $70 \%$ at baseline to 45 to $50 \%$ at Visits 5-6 and staying relatively constant $(43-46 \%)$ thereafter. Cocaine, taken by nasal insufflation, was consistently found to be the third most frequently used drug; one third of the cohort reported its use at baseline, declining to $16 \%$ by Visit 6 and staying constant (15\%) for the last 3 visits. Crack cocaine use was not assessed until Visit 12 and was reported by less than $2 \%$ of the cohort. Several major classes of drugs initially were reported used by 15 to $19 \%$ of participants, with a sharp decline to 4 to $5 \%$ by Visit 5 and stable use thereafter. These included 3,4-methylenedioxyphenylisopropylamine (MDA); psychedelics such as PCP or "angel dust," LSD, DMT, or mescaline; "downers," including barbiturates, tranquilizers, or other sedatives or hypnotics such as Quaaludes; and "uppers" such as amphetamines. The final three groups of drugs-ethyl chloride, opiates, and "other" drugswere reported used by less than $5 \%$ of the cohort at any time. 
Table 1. HIV-1 Seroconversion Rates ${ }^{a}$ by Drug Use and Sexual Behavior During 1984 to $1988^{\text {b }}$

\begin{tabular}{lllll}
\hline & \multicolumn{1}{c}{$\begin{array}{c}\text { A } \\
\text { Drug Use } \\
\text { Index }\end{array}$} & $\begin{array}{c}\text { B } \\
\text { Sexual Risk } \\
\text { Index Alone }\end{array}$ & $\begin{array}{c}\text { C } \\
\text { Sexual Risk } \\
\text { Index: No } \\
\text { Popper Use }\end{array}$ & $\begin{array}{c}\text { Dexual } \\
\text { Risk Index: } \\
\text { Popper Use }\end{array}$ \\
\hline Level 1 & $0.67(1,200)$ & $0.39(512)$ & $0.49(403)$ & $1.57(127)$ \\
Level 2 & $1.33(600)$ & $1.78(2,245)$ & $0.97(1,031)$ & $1.65(966)$ \\
Level 3 & $3.53(1,302)$ & $5.26(608)$ & $1.95(205)$ & $7.90(329)$ \\
Level 4 & $6.67(630)$ & $6.12(490)$ & $1.90(105)$ & $7.41(324)$ \\
\hline
\end{tabular}

aGiven as number of HIV-1 seroconversions per 100 person-years of observation. (See "Methods" section for the definition of each risk index.)

b Total number of person-years of observation for each behavioral category given in parentheses.

\section{Cross-sectional Associations Between Drug Use, HIV Seroconversion and Sexual Behavior Risk Levels}

The second set of analyses examined the relationships between recreational drug use and either risk of HIV seroconversion or level of RAS risk behavior. Table 1 shows the cumulative risk of seroconversion among initially seronegative men, calculated at each level of the drug use, and RAS risk indices over the course of nine semiannual visits. The risk of HIV seroconversion was several times greater for men at drug index Levels 3 or 4 than it was for men at the two lower drug use index levels. Dichotomizing the men according to whether they used multiple drugs including poppers (drug index Levels 3 and 4) or not (drug index Levels 1 and 2) revealed seroconversion rates of .5 to $1.95 / 100$ personyears for nonusers across all RAS risk levels (see Column C, Table 1) and 1.6 to 7.9/100 person-years across the four RAS risk levels (see Column D, Table 1). Overall, the average rate of seroconversion was $1.27 / 100$ person-years of observation among initially seronegative men not using poppers, and 4.55/100 person-years of observation for seronegative men reporting popper use during the prior 6 months.

Tables 2 and 3 show the serial cross-sectional association between reported use of each type of recreational substance (Table 2) or the three most common combinations of substances (Table 3 ) and high-risk RAS. Both tables illustrate the association between multiple types of recreational substances (including heavy alcohol use) and high-risk RAS, particularly at the early study assessments. However, over time only popper and cocaine use, alone or in combination with marijuana, showed a consistent cross-sectional association with RAS with multiple partners and/or without condoms.

\section{Longitudinal Associations Between Frequency of Substance Use and RAS Risk}

Changes in frequency of alcohol and drug use (marijuana, poppers, and cocaine) were analyzed for their value in predicting RAS risk at the subsequent visit using multiple logistic regression models that controlled for baseline drug 
Table 2. Summary of Significant Cross-Sectional Associations Between Substance Use Categories and High-Risk RAS, Visits 1-12

\begin{tabular}{|c|c|c|c|c|c|c|c|c|c|c|c|c|}
\hline \multirow[b]{2}{*}{ Substance:' } & \multicolumn{12}{|c|}{ Receptive Anal Sex Risk at Visit } \\
\hline & $\mathbf{1}$ & 2 & 3 & 4 & $\mathbf{5}$ & 6 & 7 & 8 & $\mathbf{9}$ & 10 & 11 & 12 \\
\hline Marijuana/Hashish & ++ & ++ & ++ & ++ & ++ & ++ & ++ & ++ & $x$ & + & $x$ & $x$ \\
\hline Poppers & ++ & $+t$ & ++ & ++ & ++ & ++ & ++ & $+t$ & ++ & ++ & ++ & ++ \\
\hline $\begin{array}{l}\text { Cocaine } \\
\text { Crack Cocaine: }\end{array}$ & ++ & ++ & $x$ & ++ & ++ & $x$ & + & $+t$ & + & $+t$ & ++ & $\begin{array}{c}++ \\
\times\end{array}$ \\
\hline $\mathrm{MDA}^{\mathrm{b}}$ & ++ & ++ & ++ & ++ & ++ & $x$ & $x$ & $x$ & $\times$ & ++ & $x$ & + \\
\hline Hallucinogens & $+t$ & ++ & $x$ & ++ & + & $x$ & $x$ & $x$ & $x$ & + & + & $x$ \\
\hline Downers & $x$ & + & $x$ & $x$ & $x$ & $x$ & $x$ & $x$ & $\times$ & $x$ & $x$ & $x$ \\
\hline Ethyl Chlorider & ++ & ++ & ++ & $+t$ & + & $x$ & $x$ & & & & & \\
\hline Heroind & $x$ & $\times$ & $x$ & $x$ & $x$ & $x$ & $x$ & $x$ & $x$ & $x$ & $x$ & \\
\hline Uppers & ++ & + & + & + & ++ & $x$ & + & ++ & $x$ & ++ & $x$ & + \\
\hline Other Drugs & $x$ & $x$ & + & $x$ & $x$ & $x$ & $x$ & $x$ & $x$ & $x$ & ++ & ++ \\
\hline Cigarettes & $x$ & $x$ & $x$ & $x$ & + & $x$ & $x$ & $x$ & $x$ & $x$ & $x$ & $x$ \\
\hline Alcohol Volume & ++ & + & $x$ & $+t$ & + & $x$ & $x$ & $x$ & $x$ & $x$ & $x$ & $x$ \\
\hline Total $N$ Per Visit & 825 & 830 & 763 & 742 & 657 & 650 & 650 & 597 & 608 & 603 & 543 & 538 \\
\hline
\end{tabular}

Note. Based on $2 \times 2$ contingency tables analyzing the use-prevalance for each substance by the incidence of risky sexual practices at each wave. Estimates of statistical significance were determined by chi-square tests. The findings from the analyses are summarized here according to the symbols: ++ : positive association and $p$ level $<.01$

$+\quad$ positive association and $p$ level $<.05$ but $>.01$

$x$ : no significant association, $p$ level $>.05$

A risk index, combining information on both number of partners for receptive anal sex (RAS) and condom usage, was constructed from the data at each wave of the Coping and Change Study. The measures for this analysis compared two risk levels: safe-respondents who, during the prior 1 -month period, had been celibate, had refrained from RAS, or had had only one partner who always wore a condom; and unsafe-monogamous individuals, who had not used condoms, or individuals reporting multiple RAS partners, regardless of condom usage. Substance-use data are from the Chicago Multicenter AIDS Cohort Study (MACS) questionnaires for the first 12 visits, spanning the years 1984 to 1990. The variables denote the percentage of men who used each substance during the preceding 6 -month period. The dichotomous alcohol measure contrasts abstemious or moderate with heavy drinking (an average of 60 or more drinks per month) over the previous 6 months.

"Not included as a separate item in the MACS interview until Visit 12.

13,4-methylenedio xyphenylisopropylamine.

- Dropped from the MACS interview after Visit 7.

"Dropped from the MACS interview after Visit 11.

use and RAS risk levels. Frequency of popper use was found to be significantly associated with subsequent sexual risk at all visits, although the other three substances were not consistently associated (data not shown). Based on those results, the sexual behavior characteristics of men reporting any popper use at each assessment were compared with the behaviors of men not reporting popper use. Men reporting popper use were consistently participating in more high-risk sexual activities than were sexually active men not reporting popper use. For example, the proportion of men engaging in RAS who did not use a condom was 1.5 to 2.0 times greater among men reporting popper use compared to nonusers $\left(P<.0001\right.$, by $\chi^{2}$ for all Visits $\left.1-9\right)$. Both monogamous men and men engaging in RAS with multiple partners showed significant differences when popper users 


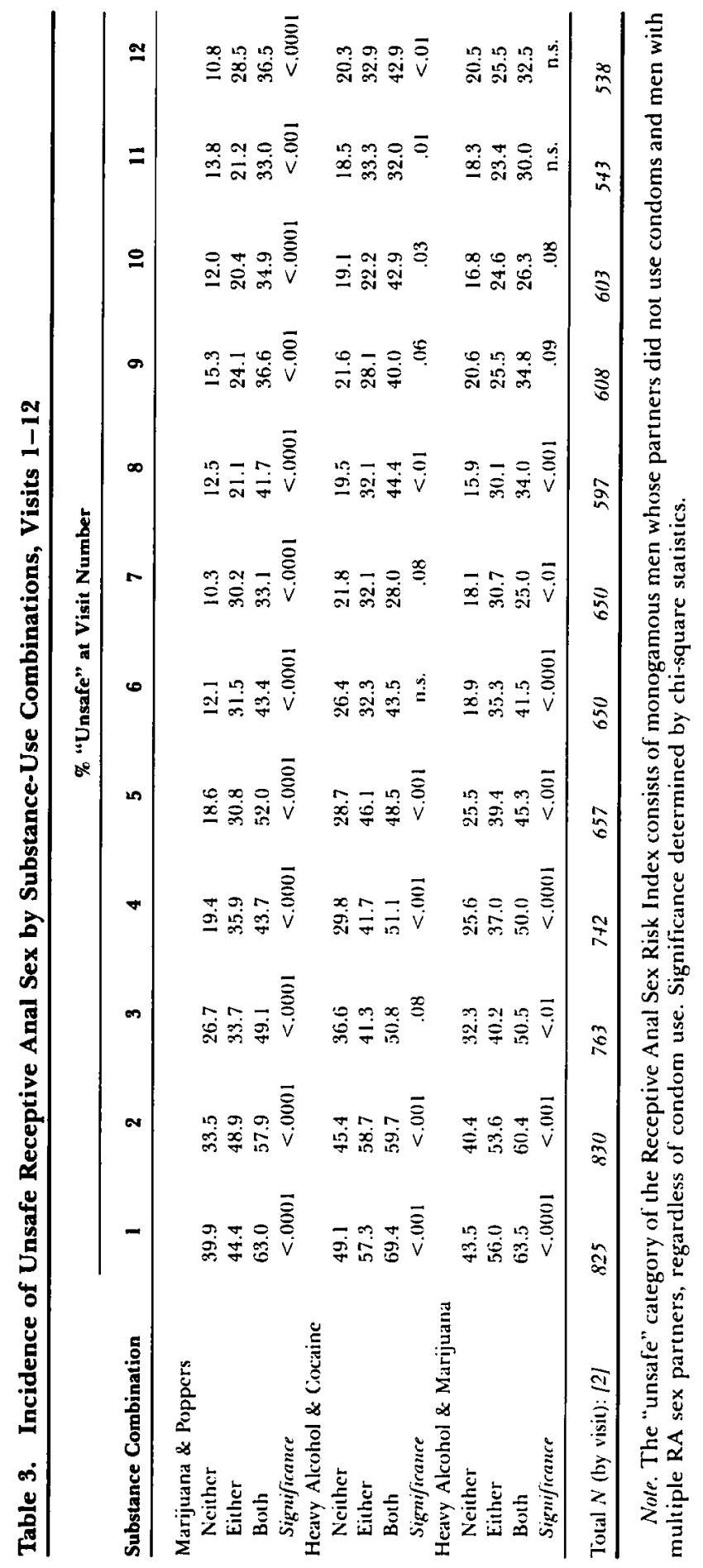


were contrasted with nonusers (data not shown). These findings, combined with earlier findings associating popper use with high-risk sex (Ostrow et al., 1990), suggest that popper use may be an important predictor of HIV-transmitting behavior in this cohort. This hypothesis was subsequently tested in the analyses described later.

\section{Prospective Associations Between Usage of Specific Recreational Drugs and Condom Use Patterns During Anal Sex}

The final set of analyses examined the association between recreational drug use and (a) the adoption of safer practices related to anal intercourse (i.e., taking up condom use or eliminating anal intercourse after reporting prior unprotected anal sex), and (2) lapse from use of safer practices (i.e., giving up condom use after prior usage). Because the practice of safer sexual behavior may differ depending on whether the person is the receptive or insertive partner in anal sex (Adib, Joseph, \& Ostrow, 1990) and whether the partners are primary or casual, these analyses examined each type of anal sex with each type of partner separately. For each of four pairs of semiannual visits for which data were collected on condom usage, the number of times participants engaged in receptive insertive anal intercourse with primary and nonprimary partners was tabulated (Visits 4-5, 5-6, 6-7, and 7-8). Within each serial pair of semiannual observations, two sets of drug and condom use behaviors were contrasted: (a) lapse in condom use in association with use of specific drugs and (b) initiation of condom use in association with cessation of drug use. Analyses were performed for each of the three most frequently used drug categories (marijuana, poppers, and cocaine) for the total group of eligible men, men in primary or monogamous relationships, and men not in primary or monogamous relationships. All of the significant findings were in the analyses that examined cessation of condom use during RAS; there were no significant findings in terms of either insertive anal sex risk change and substance use or between cessation of substance use and subsequent decreased RAS risk.

Table 4 shows the association between lapse in condom use during RAS and the use of marijuana, cocaine, and poppers. These data were analyzed for pairs of two consecutive visits. Although there was a trend toward increased risk (odds ratio) among men taking up cocaine use, the range of confidence intervals precludes drawing any conclusion about cocaine use and lapse in condom use during RAS. Only among popper users was there a statistically significant association with lapse in condom use for two of the four visit pairs and a near significant association for a third 6-month interval. Furthermore, when analyzed separately for men reporting only one primary sexual partner versus those with multiple partners, the significant association between popper use and lapse in condom use was seen only among the nonmonogamous men.

\section{DISCUSSION}

This study replicates previous findings of a cross-sectional association between high-risk male homosexual behavior and recreational drug use, and an 
Table 4. Association Between Lapsing in Recreational Drug Use and Condom Use Lapse in Receptive Anal Sex

\begin{tabular}{lccccc}
\hline Drug & Visits & $N$ & Lapsers & Odds Ratio & 95\% Confidence Interval \\
\hline Marijuana & $4-5$ & 159 & $0 / 28$ & 0.84 & $0.18-4.01$ \\
& $5-6$ & 165 & $2 / 34$ & 0.62 & $0.13-2.91$ \\
& $6-7$ & 158 & $3 / 16$ & 2.29 & $0.58-9.09$ \\
& $7-8$ & 170 & $0 / 12$ & 0.57 & $0.03-10.23$ \\
Cocaine & & & & & \\
& $4-5$ & 277 & $2 / 46$ & 0.01 & $0.21-4.75$ \\
& $5-6$ & 263 & $4 / 58$ & 0.31 & $0.40-4.27$ \\
& $6-7$ & 251 & $2 / 30$ & 1.51 & $0.31-7.23$ \\
Poppers & $7-8$ & 260 & $3 / 22$ & 18.63 & $2.93-118.41$ \\
& & & & & \\
& $4-5$ & 186 & $9 / 32$ & 5.64 & $2.07-15.35$ \\
Poppers & $5-6$ & 174 & $2 / 35$ & 0.64 & $0.14-3.01$ \\
Monogamous Men & $6-7$ & 178 & $5 / 18$ & 2.85 & $0.92-8.90$ \\
& $7-8$ & 174 & $3 / 13$ & 5.07 & $1.18-21.71$ \\
& $4-5$ & 36 & $2 / 7$ & 11.2 & \\
& $5-6$ & 36 & $0 / 12$ & 0.36 & $0.85-148.14$ \\
Poppers & $6-7$ & 32 & $0 / 5$ & 0.64 & $0.02-8.11$ \\
Nonmonogamous Men & $5-8$ & 36 & $0 / 3$ & 0.94 & $0.03-14.19$ \\
& $5-6$ & 134 & $2 / 23$ & 0.96 & $0.04-21.31$ \\
& $6-7$ & 143 & $4 / 12$ & 3.6 & $1.54-14.11$ \\
& $7-8$ & 136 & $3 / 9$ & 12.2 & $0.20-4.71$ \\
& & & & & $0.97-13.31$ \\
& & & & & $2.34-63.49$ \\
\hline
\end{tabular}

Note. For poppers, the table stratifies by sexual partner status. Monogamous men are defined as those reporting one primary sexual partner and no other partner type.

"Fraction indicates number of lapsers in condom use who also took up use of the indicated substance divided by total number of lapsers in condom use during the indicated visit-pair interval.

b Because of incomplete data on partnership status, the summation of sample sizes for the two popper subgroups do not match sizes for the overall popper group.

association between recreational drug use and continued high-risk sexual behavior (Ostrow et al., 1990). The study also demonstrates both cross-sectional and longitudinal associations between use of a specific recreational drug (i.e., poppers) and a specific sexual risk behavior (i.e., lapse in the use of condoms during anal receptive intercourse) across the entire 4.5-year observation period. The differences between these findings and those of Martin (1990), who reported a rapid decline in the magnitude of the correlation between recreational drug use and measures of unsafe sexual behavior over time in a New York City cohort, may be due, in part, to methodological differences between the two studies. The results presented here suggest that the relationship between recreational drug use and unsafe sexual behavior persisted over a 4- to 6-year period among a subgroup of men in the Chicago MACS/CCS cohort. Martin (1990) reported only parametric serial cross-sectional correlations, which became insignificant at later time periods. 
Patterns of alcohol use were relatively constant across time except for an initial reduction in heavy use at the beginning of the study, the same period when an association with increased levels of high-risk RAS was observed. Because we did not examine patterns of alcohol use change and sexual risk beyond the serial, cross-sectional analyses, no conclusions can be drawn from this study concerning possible relations between alcohol use and change in sexual behavior risk in this cohort. The proportion of men using each category of recreational drug decreased by at least $50 \%$ between 1984 and 1988, and the proportion of men abstaining from any recreational drug usage increased from none at baseline to one third of the cohort at the end of the study period. Nevertheless, approximately half of the cohort continued to use one or two recreational drugs other than poppers throughout the study period, more than $40 \%$ reported the use of poppers during the most recent assessments, and approximately $15 \%$ continued to use poppers plus one or more other drugs. The study results demonstrate that the linkage between recreational drug use and sexual risk behavior is at least partially the result of an association between patterns of use of a particular recreational drug (poppers) and lapses in condom use during RAS by nonmonogamous men. This association of popper use and unprotected RAS with casual sexual partners contributes to both lapses in condom use during RAS and the significantly higher seroconversion rate among popper users when compared to non-users.

There are several alternative explanations for the associations between recreational drug use and continued high-risk sexual behavior in prospective cohort studies of gay men (Leigh \& Stall, 1993; Stall et al., 1986). Continued drug use may prevent effective modification of sexual behavior of some homosexual men. Substance use also may be an adaptive mechanism, used to escape or deny the psychological distress caused by feeling unable to effectively change a lifethreatening behavior. Or the association may merely reflect coincident high-risk behavior that characterizes persons with impulsive or addictive personalities (Leigh, 1990). However, the multiple specific associations between popper use and HIV-transmitting behaviors and their persistence over time among a subgroup of highly educated and AIDS/HIV-knowledgeable homosexual men would argue for a direct behavioral link between popper use and unprotected $\mathrm{R} \Lambda \mathrm{S}$.

The physiological properties of volatile nitrites, in particular their ability to briefly relax the smooth muscles of the anal sphincter and thereby facilitate penetration when used during RAS, may account for the specificity of the association found in this study. However, the ability of poppers to facilitate RAS does not explain the association between their use and failure to utilize condoms during RAS, nor does it preclude other factors from contributing to the behavioral and HIV seroconversion associations found in this study. The social context for popper use among gay men, namely the historical availability of poppers at sites of sexual activity with casual or anonymous partners, might contribute to the association as well. This latter point may be important from a policy perspective because there have been both local and national efforts to ban the manufacture and sale of volatile nitrites in bars, pornographic bookstores/movie houses, bathhouses, and other sites where their use could contribute to high-risk sexual behavior. 
These findings may have important implications for the prevention of further HIV transmission among homosexual/bisexual men. Although one must caution against generalizing from the Chicago MACS/CCS cohort to the broader population of homosexually active men, it is reasonable to believe that these findings are not restricted to this cohort. The prevalence of alcohol and recreational drug use reported here closely matches that seen in other nonclinical samples of homosexual men in Chicago (McKirnan \& Peterson, 1989) and San Francisco (Stall \& Wiley, 1988). However, the very limited cross-sectional analyses of alcohol use-sexual behavior patterns in this study do not permit any insight into whether heavy alcohol use may be associated with persistent highrisk sexual behavior among some subgroups of gay men, as observed by McCusker et al. (1990).

Several recent studies, which have examined either return to unprotected anal sex (relapse) or recent HIV seroconversion among established cohorts of homosexual/bisexual men, found that recreational drug use was associated with RAS or HIV infection among educated gay men. Stall, Ekstrand, Pollack, McKusick, and Coates (1990) found that factors reported retrospectively by men in the SFMHS in 1988 who participated in unprotected RAS differed for homosexual men in a stable relationship as compared with men who reported relapse outside such relationships. For the latter men, situational factors such as being intoxicated during sex, "turned on" by partners, or unequipped with condoms were reported along with the attitudinal factors of preferring unprotected anal intercourse and perceiving peer support for taking health-related risks. Willoughby and colleagues in the Vancouver Lymphadenopathy/AIDS Cohort Study found that both higher levels of unprotected anal sex and the use of cocaine, marijuana, or amphetamines distinguished men who seroconverted from those who remained seronegative (Willoughby et al., 1990). Neither of these studies specifically implicated popper use in lapses from safe sexual behavior or in seroconversion, but they are consistent with our findings that continued high-risk sexual behavior and relapse are associated with recreational drug use. The lack of specific associations between unprotected RAS and popper use in some other cohort studies may reflect geographical differences in the popularity of drugs, smaller sample sizes, or other methodological differences with the study presented here.

The finding of a specific association between popper use and condom use relapse in RAS with nonprimary or nonmonogamous partners is potentially generalizable to at least that subgroup of homosexually active men who have begun to use condoms in response to the threat of AIDS and who are therefore at risk of lapsing to unprotected RAS and HIV infection. According to several recent studies, such occurrences could account for a significant proportion of new HIV infections among some members of the long-term cohort studies, such as the Chicago MACS/CCS (Adib, Joseph, \& Ostrow, 1990; Kingsley et al., 1991), Boston Fenway Clinic Partners Study (Seage et al., 1992) SFMHS (Stall \& Wiley, 1988), and SF Clinic Cohort Study (Lifson, 1988). In addition, poppers used simultaneously with unprotected RAS has been associated with increased relative risk of HIV infection (compared to unprotected RAS without popper use) 
among members of the Boston Fenway Clinic Partners Study (Seage et al., 1992). However, there are at least three reasons that a specific behavior or characteristic may be associated with increased rates of new infection. First, it may be because the descriptor leads to more exposure to HIV. Furthermore, this may be either because the characteristic itself directly describes such risk (e.g., nonuse of condoms) or because it serves as a surrogate for such risk (e.g., risk-taking personality style). Second, the characteristic may serve as a marker for contact with partners more likely to be infected. Certain personal characteristics (e.g., history of use of substances in settings where anonymous sexual activity with multiple partners takes place) may be associated with contact with partners who are more frequently infected. With regard to this second mechanism, it is difficult if not impossible to disentangle the separate contribution of the behavior per se from contact with HIV prevalence effects. We are uncertain whether it is what is done in places where drugs and sex are combined or whether it is the characteristics of whom one has sexual contact within the context of drug use that produce the increased risk of HIV infection. Finally, and perhaps least likely, the characteristics or behavior may be related to increased host susceptibility. In the case of alcohol or drug use, each of these may play a role and analyses presented here cannot differentiate their effects. Specifically, nonparenteral drug and alcohol use may be a marker for a style of risk taking and thus be a marker for high-risk sexual encounters. Yet it may also denote a subgroup for whom sexual partners are more frequently HIV infected so that a given behavior is more likely to produce seroconversion. Finally, it is possible that selected drugs can alter immune functioning so that exposure through unprotected RAS is more likely to produce infection (Dax, Alder, Nagel, Lange, \& Jaffe, 1991).

Thus, the messages that need to be disseminated to homosexual and bisexual men at risk of behavioral relapse are complex and potentially controversial. Just saying that recreational substance use in general may be associated with highrisk sex and is to be avoided in any situation where sexual activity is likely to take place is too simplistic, unlikely to be heeded, and potentially counterproductive. Telling persons who enjoy the intimacy and pleasure of unprotected sex that substance use can "make them unsafe" gives them an excuse to avoid taking personal responsibility for engaging in HIV-transmitting behaviors. In particular, young men beginning to experiment with homosexual behavior need to be warned of the increased risk of HIV infection through unprotected anal sex whether or not they are using recreational drugs. Behavioral intervention programs that are targeted at the combination of recreational drug use and sexual relapse among homosexual/bisexual men and increasing self-control over both types of behavior are urgently needed and, if effective, could help prevent further waves of HIV infection in those communities.

\section{REFERENCES}

Adib. M., Joseph, J., \& Ostrow, D. (1990). Relapse in safer sexual practices among homosexual men: Two year follow-up from the Chicago MACS. Program and Abstracts VIth International Conference on AIDS, 2, 262 (Abstract No. FC724). 
Calzavara, L.M., Coats, R.A., Rabound, J.M., Farewell, V.T., Read, S.E., Sheppard, F.A., Fawning, M.M., \& MacFadden, D. (1993). Ongoing high-risk sexual behaviors in relation to recreational drug use in sexual encounters. Analyses +5 years data from the Toronto Sexual Contact Study. Annals of Epidemiology, 3, 272-280.

Chmiel, J.S., Detels, R., Kaslow, R.A., VanRaden, M., Kingsley, L.A., \& Brookmeyer, R. (1987). Factors associated with prevalent human immunodeficiency virus (HIV) infection in the Multicenter AIDS Cohort Study (MACS). American Journal of Epidemiology, 126, 568-575.

Clark, W.B., \& Midanik, L. (1982). Alcohol use and alcohol problems among U.S. adults: Results of the 1979 national survey. In National Institute of Alcohol Abuse and Alcoholism. Alcoholism and health: Alcohol consumption and related problems. (Monograph No. 1) USPHS, NIAAA.

Dax, E.M., Alder, W.H., Nagel, J.E. Lange, W.R., \& Jaffe. J.H. (1991). Amyl nitrite alters human in vitro immune function. Immunopharmacology and Immunotoxicology, 13, 577-587.

Detels, R., English, P., Visscher, B.R., Jacobson, L., Kingsley, L.A., Chmiel, J.S., Dudley, J.P., Eldred, L.J., \& Kingsberg, M.H. (1989). Seroconversion, sexual activity, and condom use among 2915 HIV seronegative men followed for up to 2 years. Joumal of Acquired Immune Deficiency Syndrome, 2, 77-83.

Doll, L., Byers, R., Bolan, G., Douglas, J., Moss, P., \& Weller, P. (1989). Persistence of high-risk sexual behavior in homosexual/bisexual men: A multi-center study. Proceeds of the $V$ Intermational Conference on AIDS, Montreal, (Abstract No. MDP 23).

Gold, R.S., Skimmer, M.J., Grant, P.J., \& Plummer, C.D. (1991). Situational factors and thought processes associated with unprotected intercourse in gay men. Psychology and Health, 5, $1-$ 20.

Jaffe, H.W., Choi, K., Thomas, P.A., Havarkos, H.W., Averbach, D.M., Guinan, M.E., Rogers, M.J., Spira, T.J., Darrow, W.W., Kramer, M.A., Friedman, S.M., Monroe, J.M., Friedman-Kien, A.E., Laubenstein, C.J., Marmor, M., Safai, B., Dritz, S.K., Crispi, S.J., Fannin, S.L., Orkwis, J.P., Kelter, A., Roshing, W.E., Thacker, S.B., \& Curran, J.W. (1983). National case-control study of Kaposi's sarcoma and pneumocystis carinii pneumonia in homosexual men: Part 1. Epidemiologic results. Annals Internal Medicine, 99, 145-151.

Joseph, J.G., Emmons, C.-A., Kessler, R.C., Wortman, C.B., O'Brien, K., Hocker W.T., \& Schaefer, C. (1984). Coping with the threat of AIDS. An approach to psychosocial assessment. American Psychologist, 39, 1297-1302.

Joseph, J.G., Montgomery, S.B., Emmons, C.A., Kessler, R.C., Ostrow, D.G., Wortman, C.B., O'Brien, K., Ellcr, M., \& Eshleman, S. (1987). Magnitude and determinants of behavioral risk reduction: Longitudinal analysis of a cohort at risk for AIDS. Psychology and Health, 1, 73-96.

Kaslow, R., Ostrow, D.G., Detels, R., Phair, J.P., Polk, B.F., \& Rinaldo, C.R. (1987). The Multicenter AIDS Cohort Study (MACS): Rationale, organization and selected characteristics of the participants. American Journal of Epidemiology, 126, 310-316.

Kingsley, L.A., Zhou, S.J., Bacellar, H., Rinaldo, C.R. Jr., Chmiel, J., Detels, R., Saah, A., VanRaden, M., Ho, M., \& Munoz, A. (1991). Temporal trends in Human Immunodeficiency Virus Type 1 seroconversion 1984-1989: A report from the Multicenter AIDS Cohort Study (MACS). American Journal of Epidemiology, 134, 331-339.

Leigh, B.C. (1990). The relationship of substance use during sex to high-risk sexual behavior. Journal of Sexual Research, 27, 199-213.

Leigh, B.C., \& Stall, R. (1993). Substance use and risky sexual behavior for exposure to HIV: Issues in methodology, interpretation, and prevention. American Psychologist, 48, 1035-1045.

Lifson, A.R. (1988). Do alternate modes for transmission of human immunodeficiency virus exist? A review. Journal of the American Medical Association, 259, 1353-1356.

Martin, J.L. (1990). Drug use and unprotected anal intercourse among gay men. Heallh Psychology, 9 , $450-465$.

Martin, J.L., Dean, L., Garcia, M., \& Hall, W. (1988). The influence of drug use and lover status on gay male sexual behavior patterns. Proceeds of the IV International Conference on AIDS, Stockholm, (Abstract No. 4108).

McCusker, J., Westenhouse, J., Stoddard, A.M., Zapka, J.G., Zorn, M.W., \& Mayer, K.H. (1990). Use of drugs and alcohol by homosexually active men in relation to sexual practices. Journal of Acquired Immune Deficiency Syndrome, 3, 729-736. 
McKirnan, D.J., \& Peterson, P.L. (1989). Alcohol and drug use among homosexual men and women: Epidemiology and population characteristics. Addictive Behaviors, 14, 545-553.

Ostrow, D.G. (1989). Risk reduction for transmission of human immunodeficiency virus in high-risk communities. Psychiatric Medicine, 7, 79-95.

Ostrow, D.G., Joseph, J.G., Kessler, R.C., Soucy, J., Tal, M., Eller, M., \& Chmiel, J. (1989). Disclosure of HIV antibody status: Behavioral and mental health correlates. AIDS Education and Prevention, $1,1-11$.

Ostrow, D.G., VanRaden, M.J., Fox, R., Kingsley, L.A., Dudley, J., Kaslow, R.A., \& the MACS. (1990). Recreational drug use and sexual behavior change in a cohort of homosexual men. AIDS, 4 , $759-765$.

Penkower, L., Dew, M.A., Kingsley, L., Becker, J.T., Satz, P., Schaerf, F.W., \& Sheridan, K. (1991). Behavioral, health and psychosocial factors and risk for HIV infection among sexually active homosexual men: The Multicenter AIDS Cohort Study. American Journal of Public Health, 81 , $194-196$.

Seage, G.R. III, Mayer, K.H., Horsburgh, C.R. Jr., Holmberg, S.D., Moon, M.W., \& Lamb, G.A. (1992). The relationship between nitrite inhalants, unprotected receptive anal intercourse and the risk of HIV infection. American Journal of Epidemiology, 135, 1-11.

Sprent, P. (1989). Applied non parametric statistical methods. London: Chapman and Hall.

Stall, R., Ekstrand, M., Pollack, L., McKusick, L., \& Coates, T.J. (1990). Relapse from safer sex: The next challenge for AIDS prevention efforts. Journal of Acquired Immune Deficiency Syndrome, 3 , $1181-1187$.

Stall, R., McKusick, L., Wiley, J., Coates, T., \& Ostrow, D.G. (1986). Alcohol and drug use during sexual activity and compliance with safe sex guidelines for AIDS. Health Education Quarterly, 13, 359-371.

Stall, R., \& Wiley, J. (1988). A comparison of alcohol and drug use patterns of homosexual and heterosexual men: The San Francisco Men's Health Study. Drug Alcohol Dependence, 22, 63-73.

Weatherburn, P. (1992). Alcohol use and unsafe sexual behavior: Any. In P. Appleton, P. Davies, \& G. Hart (Eds.), AIDS: Rights, risk and reason (pp. 119-132). London: Falmer Press.

Weatherburn, P., Davies, P.M., Hickson, F.C.I., Hunt, A.J., McManus, T.J., \& Coxon, A.P.M. (1993). No connection between alcohol use and unsafe sex among gay and bisexual men $A I D S, 7$, $115-119$.

Willoughby, B.C., Schechtor, M.T., Craib, K.J.P., McLeod, W.A., Douglas, B., Fayk, S., Nitz, R., \& O'Shaughnessy, M. (1990). Characteristics of recent seroconverters in a cohort of homosexual men: Who are the prevention failures? Final Program and Abstracts Sixth International Conference on AIDS, 2, 101 (Abstract No. FC45). 